\title{
The relation between visual imagery mediators and recall
}

\author{
JOHN C. DAY and FRANCIS S. BELLEZZA \\ Ohio University, Athens, Ohio 45701
}

\begin{abstract}
Three hypotheses are discussed as explanations for the result that pairs of concrete nouns are more easily remembered than are pairs of abstract nouns: the imagery hypothesis, the familiarity hypothesis, and the concreteness hypothesis. Two experiments are reported in which the degree of visual imagery associated with the components of paired associate items was not indicative of the degree of visual imagery experienced during their learning or with the accuracy with which they were recalled. It was found that pairs of related abstract nouns were rated higher in imagery and familiarity than were pairs of unrelated concrete nouns, but recall of the higher imagery pairs was poorer. The concreteness hypothesis is discussed as the best explanation for the results. The concreteness hypothesis proposes that people learn to associate the labels of concrete objects by using their real-world knowledge of the potential relations between categories of objects. Dual coding theory and schema theory are also discussed as explanations for mediation learning, and the issue of visual imagery as an epiphenomenon is addressed.
\end{abstract}

The study of visual imagery is an important aspect of contemporary cognitive psychology. Imagery seems to play an important role in the manner in which information is represented in memory (Kosslyn, 1980; Paivio, $1971,1977,1978$ ) and in the manner in which new information is remembered. Recent discussion of visual imagery has focused primarily on the representational characteristics of imagery (J. R. Anderson, 1978; Kosslyn \& Pomerantz, 1977; Paivio, 1977, 1978; Pylyshyn, 1973).

In addition, people can use visual imagery mediation as a strategy to associate information in memory (Bower, 1972; Paivio, 1969, 1971). For example, when presented the noun pair "truck-apple," a person can use the learning strategy of forming a visual image of a truck running over an apple. Later, when only the cue, "truck," is presented, the image previously formed can be retrieved from memory and decoded into the correct response, "apple." Formation of a visual image of the referents of a pair interacting in some way is the reason sometimes given for why learning occurs. Visual imagery as the cause of learning will be referred to here as the imagery hypothesis. Hence, visual imagery is important both as a model of representing information in memory and as a strategy for learning new information. The purpose of the experiments reported here was to look

The authors would like to thank Susan M. Conners and Myriam Ruthchild for their help in collecting the data in Experiment 1. Thanks go also to the Ohio University Computer and Learning Services for making computer time and facilities available. This research was supported in part by a grant to the second author by the Field-Wiltsie Foundation. Requests for reprints may be sent to Francis S. Bellezza, Department of Psychology, Ohio University, Athens, Ohio 45701. more closely at the nature of learning using visual imagery mediation.

Although visual imagery has come to play an important role in theories of mediated learning, there are situations in which the amount of visual imagery reported by subjects is not indicative of the degree of learning that takes place. In a series of experiments, Bellezza and Day (Note 1) demonstrated that both letters of the alphabet and nouns representing concrete objects are rated high in visual imagery. However, when composite images were formed for pairs of letters and concrete nouns in a paired associate learning task, the imagery ratings for the letter-noun pairs were significantly lower than the ratings for the noun-noun pairs. Furthermore, the letter-noun pairs were not recalled as well as the noun-noun pairs. These results show that the imagery ratings of the individual components of a pair do not always indicate the degree of imagery experienced when the components are formed into a composite image.

\section{The Familiarity Hypothesis and}

the Concreteness Hypothesis

The imagery hypothesis indicates that the formation of a vivid composite image will result in good performance in a paired associate learning task. Also, it implies that high-imagery components will result in high imagery for the presented pair (Bower, 1972; Paivio, 1971). Consequently, the imagery hypothesis does not seem to be able to account for the results of Bellezza and Day (Note 1). Since the letters and nouns used by Bellezza and Day were rated equally high in imagery, subjects should have been able to form interacting images using them.

A possible explanation for the low levels of learning that occurred in the Bellezza and Day (Note 1) experi- 
ments is that people have little prior experience of letters of the alphabet interacting with physical objects. Therefore, associating letters and objects is difficult. Little prior knowledge exists in memory that involves physical objects interacting with letters of the alphabet, yet this interaction seems to be necessary for learning by visual imagery mediation (Bower, 1972; Wollen, Weber, \& Lowry, 1972). This is the familiarity hypothesis. It suggests that learning by visual imagery mediation makes use of composite images based on the learner's experience. The letters of the alphabet are simply visual symbols. Therefore, composite visual images involving letters and physical objects interacting are difficult to form, and the pairs are difficult to remember. Recall of a letter and a physical object is not likely to be redintegrative (Horowitz \& Prytulak, 1969) in the sense that the two items in the image cannot be represented by a single event in memory. When presented with an easily learned word pair such as "clown-dog," a person might remember a specific experience of a clown and a dog doing tricks together at a circus. By remembering this specific episode, the learner retrieves sensory-perceptual information connected with the episode and thereby experiences visual imagery. The visual imagery indicates that information is available in memory that can relate the two words in the presented pair.

The familiarity hypothesis, however, does not seem to be able to account for all mediated learning and its accompanying visual imagery. For example, many people can form a composite image for the word pair "Eskimo-dinosaur." Yet, few people think about an Eskimo and a dinosaur together until the pair is presented. To form a visual image, it seems likely that people first retrieve from memory some of the classes and categories of objects to which Eskimo and dinosaur belong. For example, an Eskimo belongs to the more general category "man," and a dinosaur belongs to the category "large animal." The generic knowledge regarding men and large animals and the sensory-perceptual information specific to Eskimos and dinosaurs enable the person to form a visual image. This is the concreteness hypothesis. It proposes that learning by visual imagery mediation is based on general knowledge of how classes of concrete objects interact in the physical world. A man may ride a large animal, a man may kill a large animal, a man may be trampled by a large animal, a man may feed a large animal, and so on. The referents of concrete nouns have attributes that are often compatible with each other. Hence, the composite visual image formed is dependent on knowledge about how classes of concrete objects interact in the physical world and is not dependent on personal experience with the two specific objects interacting.

The purpose of Experiment 1 was to try to manipulate both the factors of familiarity of word pairs (relatedness) and their concreteness. A determination was then made of the degree to which each of these two factors contribute to imagery ratings and recall performance.

\section{EXPERIMENT 1}

In Experiment 1, both abstract- and concrete-noun pairs were used. In this manner, the factor of concreteness was manipulated. In addition, half of the pairs used contained nouns judged to be related and the other half contained unrelated nouns. It was expected that this factor of relatedness would indicate the degree to which the composite images formed for the pairs would be familiar or unfamiliar. If concreteness is the primary factor in learning, then the only result should be that concrete-noun pairs are recalled better than abstractnoun pairs. If familiarity is the primary factor in learning, then the only result should be that the related pairs are recalled better than the unrelated pairs. These two alternative outcomes represent ideal solutions to the problem. A more likely result is that both factors are significant to some degree. Therefore, one of the statistical analyses performed in Experiment 1 was to compute a ratio between the amount of variance in the recall measure accounted for by the two factors of concreteness and familiarity.

To gather additional evidence about the degree to which subjects were drawing upon prior experience when imaging and learning the pairs, the subjects described the composite image formed for each pair, the image's vividness and clarity were rated, its familiarity was rated, and, finally, the subject had to indicate where he or she thought the image originated. Was it purely imaginary, or was it based on a picture in a book, a magazine, a movie, a TV show, and so on? It was hoped that these descriptive data would provide some insights about the degree to which the images formed were based on the relations between the categories to which the words belonged and on the specific experiences of which the subjects were reminded.

\section{Method}

Subjects. A total of 71 students enrolled in introductory psychology courses at Ohio University volunteered to participate for extra course credit.

Materials. Twenty-four word pairs were made up such that 12 of the pairs contained nouns representing concrete objects and 12 of the pairs contained nouns that were abstract. Furthermore, six pairs of each type were made up of seemingly unrelated nouns (brain-pipe, happiness-opinion) and six pairs of each type were made up of seemingly related nouns (student-book, joy-victory). All the words used occurred at least 50 times per million in the Thorndike and Lorge (1944) general frequency count. The imagery ratings (Paivio, Yuille, \& Madigan, 1968) for the abstract nouns ranged from 2.13 to 5.60 , and those for the concrete nouns ranged from 5.93 to 6.73 .

Procedure. In the first part of the experiment, a group of 44 subjects rated the relatedness of the two nouns in each of the 24 pairs using a 5-point rating scale in which " 5 " meant that the words were very related and " 1 " meant that the words were very unrelated. Subjects were told to rate these pairs on the basis of how closely related the meaning of one word was to the meaning of the other. A 2 by 2 analysis of variance was performed on the mean relatedness ratings provided for each type of pair by each of the 44 subjects. The two factors were word concreteness and word relatedness, and both were within- 
subjects factors. As expected, pairs intended to be unrelated were rated lower than pairs intended to be related $[F(1,43)=$ $773.84, \mathrm{p}<.001, \mathrm{MSc}=.379]$. The mean ratings were 1.34 and 3.92 , respectively. Also, abstract words were generally rated as somewhat more related than concrete words $[\Gamma(1,43)=8.97$. $\mathrm{p}<.005$, MSe $=.107]$, with means of 2.70 and 2.55 , respectively. These 'results indicated that people perceived the unrelated pairs as made up of unrelated nouns and the related pairs as made up of related nouns.

In the second part of the experiment, 27 new subjects were tested individually. The noun pairs were presented one at a time on an index card, and the subject was to try to form a composite visual image using the referent images of the words or images that were associated with each word. The subject was also to describe the composite image formed in one or two sentences. Next, the clarity of the composite image was rated by the subject using a 5-point rating scale on which " 5 " meant that the image was very clear and vivid and "l" meant that no image was present. The subject also rated the familiarity of the composite image using a 5-point scale of familiarity on which " 5 " meant that the image formed was very familiar and " 1 " meant that the image formed was completely unfamiliar to the subject or that no image was present. If the image was not entirely new, the subject was asked to indicate where he or she had experienced that image before.

Each sequence of four pairs contained one of the four types of pairs in a random order. After all 24 pairs had been presented, the subjects were given a surprise cued recall test. The first word of each pair was presented in the same order as the pairs were, and the subject was to try to recall the second word of the pair. Both the presentation and the testing of the pairs were self-paced.

\section{Results}

A 2 by 2 analysis of variance was performed on the mean imagery ratings, on the mean familiarity ratings, and on the arcsine transformations of the proportion of words correctly recalled. The two factors were again noun concreteness and noun relatedness, with both factors being within-subjects factors. The mean value of each variable for each of the four types of word pairs is shown in Table 1.

The analysis of the imagery ratings for the composite images showed both noun concreteness $[F(1,26)=$ $12.43, \mathrm{p}<.005, \mathrm{MSe}=.164]$ and noun relatedness $[F(1,26)=44.81, p<.001, \mathrm{MSe}=.275]$ to be significant factors. Concrete-noun pairs were rated as higher in imagery (4.25) than were abstract-noun pairs (3.98), and related word pairs (4.45) were also rated higher in imagery than were unrelated word pairs (3.77). An

Table 1

Mean Imagery Ratings, Mean Familiarity Ratings, and Mean Proportion Recalled for the Four Types of Pairs Used in Experiment 1

\begin{tabular}{lrrrr} 
& \multicolumn{4}{c}{ Item Type } \\
\cline { 2 - 5 } Measure & $\mathrm{AR}$ & $\mathrm{AU}$ & $\mathrm{CR}$ & $\mathrm{CU}$ \\
\hline Imagery & 4.27 & 3.68 & 4.63 & 3.87 \\
Familiarity & 4.00 & 3.64 & 4.30 & 3.09 \\
Recall & .55 & .43 & .66 & .61 \\
\hline
\end{tabular}

Note $-A R=$ abstract nouns, related: $A U=$ abstract nouns, unrelated $C R=$ concrete nouns, related; $C U=$ concrete nouns, unrelated. additional unexpected result was that related abstract nouns (4.27) were rated significantly higher in imagery than were unrelated concrete nouns $(3.87)[F(1,26)=$ $8.73, \mathrm{p}<.01, \mathrm{MSe}=.249]$. As in the experiments reported by Bellezza and Day (Note 1), the imagery ratings of the composite images were not determined solely by the imagery ratings of their components.

The analysis of variance on the familiarity ratings of the composite images showed noun relatedness to be a significant factor $[F(1,26)=62.20, \mathrm{p}<.001, \mathrm{MSe}=$ .267], but not noun concreteness. The images formed from related nouns were rated 4.15 on the 5-point familiarity scale, and the unrelated nouns, 3.37. However, the Noun Concreteness by Noun Relatedness interaction was also significant $[F(1,26)=19.70, p<.001$, $\mathrm{MSe}=.256]$. As in the case of the imagery ratings, the mean familiarity rating for pairs of related abstract nouns was significantly greater than the mean familiarity rating for pairs of unrelated concrete nouns $[F(1,26)=44.02, p<.001, \mathrm{MSe}=.256]$.

Analysis of the transformed proportions of response words correctly recalled showed noun concreteness to be significant $[F(1,26)=36.87, \mathrm{p}<.001, \mathrm{MSe}=.058]$. For the concrete pairs, .64 of the response words were correctly recalled, whereas for the abstract pairs, the proportion was .49 . Noun relatedness was also significant $[F(1,26)=26.28, p<.001, \mathrm{MSe}=.028]$. Of the related pairs, .60 were correctly recalled, and of the unrelated pairs, .52. The Noun Concreteness by Noun Relatedness interaction was also significant $[F(1,26)=$ $4.90, \mathrm{p}<.05, \mathrm{MSe}=.023$ ]. Somewhat surprisingly, the unrelated concrete pairs were recalled better $(.61)$ than were the related abstract nouns $(.55)[F(1,26)=$ $8.00, \mathrm{p}<.01, \mathrm{MSe}=.023]$, even though the related abstract pairs were rated significantly higher in imagery and familiarity.

\section{Discussion}

The results of Experiment 1 produced an unexpected pattern of means on the imagery, familiarity, and recall measures. The most interesting result was that the imagery and familiarity ratings for related abstract pairs were significantly higher than those for unrelated concrete pairs. However, for the recall measure, just the opposite effect was found. The unrelated concrete pairs were recalled better. This result seems to separate recall performance both from the amount of imagery experienced during learning and from the amount of specific episodic knowledge drawn upon during learning. One might expect that those pairs rated highest in imagery would be recalled best, but this did not occur. Pairs whose composite images were rated as most familiar were not best recalled either. The factor that was the best indicator of learning was noun concreteness. Hence, the results of Experiment 1 support the concreteness hypothesis more than they support either the imagery hypothesis or the familiarity hypothesis. Although 
pairs of words representing concrete objects resulted in neither the most imagery nor the most familiar images, they were learned the best. The basis for learning under the concreteness hypothesis seems to be that people have knowledge about how classes of objects in the physical world can relate to each other. This knowledge is used as a basis for learning pairs of words representing concrete objects, even though the specific objects may not have been experienced together before the experiment.

Although a number of significant interactions were found in Experiment 1, the main effects of noun concreteness and noun relatedness affected the visual imagery ratings and the proportion of words recalled. Therefore, the effects of these two independent variables were compared in the manner planned before the experiment. To obtain an indication of the relative effects of these two factors on the imagery ratings, the ratio of the variance of the imagery ratings accounted for by the two factors was found by computing the ratio of their variance component values (Kirk, 1968). The ratio of noun relatedness variance over noun concreteness variance was 6.44 . This indicates that noun relatedness was more of a determinant of vividness of imagery than was noun concreteness.

The same ratio was computed for the ratings representing the familiarity of the images. In this case, the ratio was 96.10 , indicating that noun relatedness accounted for 96.10 times the variance accounted for by noun concreteness. In fact, the factor of noun concreteness was not a significant determinant of the familiarity ratings. However, interpretation of these results is difficult, as mentioned above, because the Noun Relatedness by Noun Concreteness interaction was significant.

When the recall results were analyzed, both noun relatedness and noun concreteness were significant factors. However, the amount of variance accounted for by concreteness was about three times that accounted for by relatedness. This result is the opposite of the results found for the measures of image vividness and image familiarity. Noun concreteness accounts for more recall variance than does noun relatedness. Again, however, the significant Noun Concreteness by Noun Relatedness interaction makes any generalization about main effects difficult.

During presentation of the pairs, subjects indicated that they recognized a mean of .93 of the images they formed. The other .07 were labeled as imaginary. They described .51 as being based on personal experiences, .23 based on TV and movies, .08 based on magazines and books, and .11 from other sources. This result supports the notion that visual images represent retrieved sensory-perceptual information that has been previously experienced and stored in memory. Also, subjects seemed to be able to indicate the source of these images. The value of .93 is larger than would be expected if subjects were using their imagination to create images of interacting nouns that were rated as unrelated. However, there is no way to determine whether the subjects were rating the familiarity of the composite image or were merely rating the familiarity of the images of the individual components, such as "Eskimo" and "dinosaur."

\section{EXPERIMENT 2}

Experiment 1 provided some evidence showing that degree of visual imagery experienced during learning may not be indicative of the amount learned. However, Experiment 1 may be criticized for some methodological reasons. Only six pairs of each type of pair were used. Also, the imagery rating task was embedded in a series of other tasks involving ratings of image familiarity, image description, and image identification. The purpose of Experiment 2 was to use a broader range of materials and a simpler procedure to replicate the most important result of Experiment 1, that is, the result that the related abstract noun pairs were rated higher in imagery but recalled more poorly than were the unrelated concrete nouns.

\section{Method}

Subjects. Eighty-three students enrolled in introductory psychology courses at Ohio University volunteered to participate for extra course credit.

Materials. Sixty-four abstract nouns were selected from the Toglia and Battig (1978) norms, and 32 pairs were formed such that the two words in each pair were judged by the experimenters to be highly related (democracy-liberty). Another 64 nouns were selected from the same source and consisted of concrete nouns that were formed into 32 pairs that were judged to be unrelated (cheese-fur). Pairs of words synonymous in meaning were not used. Abstractness was defined as a rating less than 4.00 on the 7-point concreteness scale of Toglia and Battig (1978), and concreteness was defined as a rating greater than 6.00. All the words were highly meaningful, with a rating greater than 4.00 on the Toglia and Battig 7-point meaningfulness scale.

A group of 42 subjects was used to obtain relatedness ratings for the 64 pairs of words. The pairs were randomly ordered into a list, and the subjects were given $10 \mathrm{sec}$ to rate each pair using a 7-point relatedness scale on which " 7 " indicated that the words were very much related in meaning and " 1 " indicated that the words were completely unrelated in meaning. The mean relatedness rating for each pair was then computed. The 16 most related abstract pairs and the 16 least related concrete pairs were selected for further use. The abstract pairs ranged in relatedness from 5.50 to 6.50 , and the concrete pairs ranged from 1.19 to 1.93 .

In addition to these 32 pairs of words, 16 letters were randomly selected from the alphabet and paired with 16 concrete nouns not used for the noun pairs. These letter-noun pairs were used to try to replicate the results of Bellezza and Day (Note 1), which indicated that letter and concrete noun pairs would be rated lower in imagery and be more poorly recalled than pairs made up of concrete nouns. All 48 pairs of items were randomly placed in a list in sets of 3 pairs, with each set containing one of each of the three types of pairs (related abstract, unrelated concrete, letter-noun) in random order. 
Procedure. In the first part of the main experiment, 41 subjects were shown the list of 48 pairs at a rate of one pair every $10 \mathrm{sec}$ and were instructed to form an interacting visual image of the two items in each pair. After forming an image, subjects rated the clarity and vividness of that composite image using a 7-point imagery scale on which " 7 " represented an image that was very clear and vivid and " 1 " indicated that no image could be formed. The experimenter gave example images for a related abstract pair and a letter-noun pair. The related abstract pair was "joy-victory," and the image described consisted of fans at a basketball game jumping for joy when their team won a victory. The letter-noun pair used was "A-dog," and the image described was a dog chewing on the leg of the capital letter A. Subjects were also instructed to remember each pairing because they would have to recall the second word from each pair when shown the first item later on.

A 1-min distractor task followed the presentation of the pairs, and then the subjects were tested using the first item from each pair. These items were again randomly placed in the list in groups of three, with a random ordering of the three types of pairs in each group. The list of cue items was printed on a sheet of paper, and the subjects were paced through this list at a rate of one cue every $10 \mathrm{sec}$. After going through the list once, subjects were given extra time to go back and recall any response words they may have missed.

\section{Results}

A one-way analysis of variance was performed on the mean imagery ratings for the three types of pairs (related abstract, unrelated concrete, letter-noun). The mean ratings are shown in Table 2 . There was a significant difference in the imagery ratings for the three types of pairs $[\mathrm{F}(2,80)=61.51, \mathrm{p}<.001, \mathrm{MSe}=.828]$. Related abstract pairs were rated higher in imagery (5.94) than were either unrelated concrete-noun pairs (4.15) or letter-noun pairs (3.88). Two a priori contrasts were tested. The first constrast showed that the mean imagery rating for the composite images of related abstract pairs was significantly greater than that for unrelated concrete pairs $[\mathrm{F}(1,80)=37.38, \mathrm{p}<.001, \mathrm{MSe}=.828]$. The second contrast showed that the composite images of unrelated concrete pairs were rated as significantly higher in imagery than those for letter-noun pairs $[\mathrm{F}(1,80)=6.89, \mathrm{p}<.025, \mathrm{MSe}=.828]$.

A second one-way analysis of variance was performed on the mean proportion of response words recalled from the three types of pairs. These mean proportions are shown in Table 2. Recall for the three types of pairs was significantly different $[F(2,80)=72.44$, $\mathrm{p}<.001, \mathrm{MSe}=.020]$. Unrelated concrete pairs were

Table 2

Mean Imagery Ratings and Proportion of Items Recalled for the Three Types of Pairs Used in Experiment 2

\begin{tabular}{lrrr} 
& \multicolumn{3}{c}{ Pair Type } \\
\cline { 2 - 4 } & $\mathrm{LC}$ & $\mathrm{CU}$ & $\mathrm{AR}$ \\
\hline Imagery Rating & 3.88 & 4.15 & 5.94 \\
Proportion Recalled & .30 & .67 & .57 \\
\hline
\end{tabular}

Note-LC = pairs of letters and concrete nouns; $C U=$ pairs of unrelated concrete nouns; $A R=$ pairs of related abstract nouns. recalled more often (.67) than related abstract pairs (.57), and these items were recalled more often than letter-noun pairs (.30). Again, two a priori contrasts were tested. The first contrast showed that unrelated concrete pairs were recalled more often than related abstract pairs $[\mathrm{F}(1,80)=102.7, \mathrm{p}<.001, \mathrm{MSe}=.020]$. The second contrast showed that related abstract pairs were recalled more often than letter-noun pairs $[F(1,80)$ $=380.13, \mathrm{p}<.001, \mathrm{MSe}=.020]$.

\section{Discussion}

The results of Experiment 2 confirm that word concreteness is an important indicator of success in paired associate learning. Pairs of unrelated concrete nouns were recalled better than were pairs of related abstract nouns. These, in turn, were recalled better than pairs made up of letters and concrete nouns. However, the imagery ratings of the composite images for the related abstract nouns were significantly greater than those of the unrelated concrete nouns. These results support the concreteness hypothesis more than they do the familiarity hypothesis or the imagery hypothesis. Pairs of related abstract words were previously judged as related in meaning. That is, the relation between the two words was judged as familiar. But these pairs were not recalled as well as the pairs of concrete nouns judged as unrelated. Similarly, the pairs of related abstract nouns were judged as producing the greatest amount of visual imagery, but they were not recalled as well as the pairs of unrelated concrete nouns that were rated lower in visual imagery. As was reported in Experiment 1, familiarity and degree of imagery may be factors in learning pairs of verbal units, but the concreteness of the objects depicted seems to be the most important factor.

\section{GENERAL DISCUSSION}

The relation between visual imagery and learning seems to be a strong one (Paivio, 1971), and the purpose of the experiments presented here was to explore some of the reasons for this relationship. However, results were obtained that show that experienced visual imagery is not always indicative of the amount learned. Our results seem to indicate that visual imagery is best thought of not as a causal factor in learning, but rather as sensory-perceptual information accompanying the other information retrieved from memory by the presented pair. This imagery may or may not be indicative of how well the presented item will be retained. Furthermore, the prior knowledge upon which the image is based may be a memory of some specific experienced event as when one remembers a circus act when presented with the pair "clown-dog." But this episodic information does not seem to be necessary for learning. Learning may also be based on generic knowledge regarding the classes or categories to which the physical objects named in the pair belong. Presentation of the 
pair "Eskimo-dinosaur" can result in information being retrieved from memory about the categories "man" and "large animals" and how they may interact. Experiments 1 and 2 showed that pairs of unrelated object labels may not generate as much imagery as other types of pairs, but they may be better recalled.

One could argue, of course, that visual imagery is merely an epiphenomenon accompanying learning and has nothing functional to do with it (Pylyshyn, 1973; Ryle, 1949). The evidence presented here provides support for this position. The presence of visual imagery is not the reason that learning occurs. However, visual imagery can sometimes indicate that learning is taking place and suggest why it is taking place. Furthermore, a distinction can be made between associated imagery and schematic imagery. It is possible that a pair of related abstract words such as "joy-victory" readily elicits sensory-perceptual information from memory associated with the presented pair. This image could consist of fans jumping to their feet in joy after their team won a victory. However, the abstract information presented in the pair is not represented in the experienced image because the words are abstract. This type of imagery can be characterized only as associated imagery. The image does not closely represent the information presented in the pair. When it is recalled, the information in an associated image cannot be easily decoded into the original pair of words (Paivio, 1971). When presented a pair of unrelated concrete words such as "brain-pipe," the image created may be less vivid and less likely to be based on personal experience. But the learner may be able to use general knowledge relating parts of the body to physical objects and think of the relation "physical injury to part of body (brain) caused by physical object (pipe)." Any image formed for this relation is a schematic image because components of the image are isomorphic to the objects denoted by the noun pair. The image can be decoded when later recalled. This schematic image, however, may be rated as not as clear and vivid as an associated image.

This emphasis on concreteness independent of imageability can also help explain the results of Jonides, Kahn, and Rozin (1975), who found that imagery instructions improved paired associate performance in congenitally and totally blind subjects. Their results can be better understood if it is assumed that blind subjects have haptic experience with concrete objects interacting in various ways, although they may not have visual images of them. Their memory of the objects is based on touching and kinesthetic experiences. The imagery instructions the blind subjects received during the experiment may have encouraged them to draw upon their real-world knowledge during learning, although this knowledge was not gained through the visual modality.

\section{Dual Coding Theory}

The account of mediated learning based on realworld knowledge proposed here may be contrasted to
Paivio's (1971, 1977, 1978) dual coding theory. In dual coding theory, abstract nouns are represented only in the linguistic-verbal system, whereas concrete nouns are represented both in the verbal-linguistic and in the pictorial-imagery system. In dual coding theory, concrete nouns are learned better than abstract nouns because they become associated in both memory systems, whereas abstract noun pairs become associated only in the linguistic-verbal system.

Dual coding theory cannot easily explain the results presented here and by Bellezza and Day (Note 1). Bellezza and Day found that both letters and words were rated high in visual imagery, but the letter-noun pairs were not rated high in imagery and also were not remembered as well as the concrete-noun pairs. This result supports the notion that the mode by which individual items are represented in memory does not indicate ease of learning. Real-world knowledge of possible relations between items in a pair is more important than their mode of representation in memory.

Using dual coding theory, it is also difficult to explain why pairs of related abstract nouns elicit greater visual imagery than do pairs of unrelated concrete nouns. Again, the emphasis in dual coding theory is on the nature of the representation of individual nouns, and the theory can account for neither the imagery nor the recall performance found here. Because of the importance in mediated learning of potential relations among components, the traditional method of using the meaning. fulness, discriminability, or imagery attributes of individual verbal items to predict ease of learning may be limited in usefulness. If any one attribute of individual nouns is important in predicting recall performance, it is concreteness. When pairs are made up of concrete nouns, there is a good chance that the learner has some knowledge of a relation between the classes of the physical objects represented by the two nouns. This knowledge can then be used as mediating information to associate the pair.

\section{Schema Theory}

The type of learning that has been discussed here is called mediation learning because the learner is instructed to use prior knowledge as a means of connecting the items in the pair (Montague, 1972). The learner is sometimes aware of accessing this prior knowledge because he or she experiences visual images or verbal phrases combining the items presented. However, as argued above, these experiences may accompany learning but not be the cause of it. The problem remains of identifying the cognitive mechanisms that underlie mediation learning. In recent years, the notion of memory schemata has begun to develop and play a role in memory theory Memory schemata are organized generic knowledge structures that can influence the comprehension and retention of new information (R. C. Anderson, 1979; Bobrow \& Norman, 1975; Neisser, 1976; Rumelhart, 1980; Rumelhart \& Ortony, 1977; Schank, 1980; 
Schank \& Abelson, 1977). Therefore, schema theory provides a potential explanation of the mediation process in paired associate learning.

Noun concreteness also seems to be important in schema theory. Concepts representing concrete objects can be bound as variable values in the slots of memory schemata. In almost all the examples provided of variable values bound by schemata, these variable values represent concrete objects. Rumelhart and Ortony (1977) define memory schemata as based on real-world knowledge and not on verbal definitions. Schemata represent the arrangement of things and not of words, and schemata themselves are not linguistic. As an example, Rumelhart and Ortony discuss the schema for the concept "give" as needing variable values representing the gift, the giver, and the recipient. In a similar manner, Schank and Abelson (1977) note that the restaurant script needs values for props such as foods, drinks, and the objects found in a restaurant. Also, specific people are needed for the roles of hostess, waiter, customer, and so on. Perhaps some of the emphasis in schema theory on relations among real-world objects results from their use in discussing perception (Minsky, 1975; Palmer, 1975). For whatever reason, memory schemata have been used primarily to represent objects, persons, situations, events, sequences of events, actions, and sequences of actions. Schemata are concepts but do not represent relations among words or concepts. Hence, memory schemata cannot easily be used to relate and store pairs of related abstract nouns, such as those presented in Experiments 1 and 2, and cannot easily be used to learn the pairs of letters and concrete nouns presented in Experiment 2 and by Bellezza and Day (Note 1). Unlike dual coding theory, schema theory emphasizes the importance of relations among objects in the physical world rather than their mode of representation in memory.

\section{REFERENCE NOTE}

Bellezza, F. S., \& Day, J. C. The alphabet mnemonic and visual imagery mediation. Paper presented at the annual meeting of the Psychonomic Society, Philadelphia, Penn, November 1981.

\section{REFERENCES}

Anderson, J. R. Arguments concerning representations for mental imagery. Psychological Review, 1978, 85, 249-277.

Anderson, R. C. Schema-directed processes in language comprehension. In A. Lesgold, J. Pellegrino, S. Fokkema, \& R. Glaser (Eds.), Cognitive psychology and instruction. New York: Plenum, 1979.

Bozrow, D. G., \& Norman, D. A. Some principles of memory schemata. In D. G. Bobrow \& A. Collins (Eds.), Representation and understanding. New York: Academic Press, 1975.
Bower, G. H. Mental imagery and associative learning. In L. Gregg (Ed.), Cognition in learning and memory. New York: Wiley, 1972.

Horowitz, L. M., \& Prytulak, L. S. Redintegrative memory. Psychological Review, 1969, 76, 519-531.

Jonides, J., KAHN, R., \& RozIN, P. Imagery instructions improve memory in blind subjects. Bulletin of the Psychonomic Society, $1975,5,424-426$.

KınK, R. E. Experimental design procedures for the behavioral sciences. Belmont, Calif: Wadsworth, 1968.

Kosslyn, S. M. Image and Mind. Cambridge, Mass: Harvard University Press, 1980.

Kosslyn, S. M., \& Pomerantz, J. R. Imagery, propositions, and the form of internal representations. Cognitive Psychology, $1977,9,52-76$.

Minsky, M. A framework for representing knowledge. In P. Winston (Ed.), The psychology of computer vision. New York: McGraw-Hill, 1975.

Montague, W. F. Elaborative strategies in verbal learning and memory. In G. H. Bower (Ed.), The psychology of learning and motivation (Vol. 6), New York: Academic Press, 1972.

Neisser, U. Cognition and reality. San Francisco: Freeman, 1976.

Paivio, A. Mental imagery in associative learning. Psychological Review, 1969, 76, 241-263.

Paivio, A. Imagery and verbal processes. New York: Holt, Rinehart, \& Winston, 1971.

PaIvio, A. Images, propositions, and knowledge. In J. M. Nichols (Ed.), Images. perception, and knowledge. Boston: D. Reidel Publishing, 1977.

Paivio, A. A dual coding approach to perception and cognition. In H. L. Pick, Jr., \& E. Saltzman (Eds.), Models of perceiving and processing information. Hillsdale, N.J: Erlbaum, 1978.

Paivio, A., Yuille, J. C., \& Madigan, S. A. Concreteness, imagery, and meaningfulness values for 925 nouns. Journal of Experimental Psychology Monograph, 1968, 76 (1, Pt. 2).

Palmer, S. E. Visual perception and world knowledge: Notes on a model of sensory-cognitive interaction. In D. A. Norman \& D. E. Rumelhart (Eds.), Explorations in cognition. San Francisco: Freeman, 1975.

Pylyshyn, Z. W. What the mind's eye tells the mind's brain: A critique of mental imagery. Psychological Bulletin, 1973, 80, 1-24

RUMelhart, D. E. Schemata: The building blocks of cognition. In R. Spiro, B. Bruce, \& W. Brewer (Eds.), Theoretical issues in reading comprehension. Hillsdale, N.J: Erlbaum, 1980.

Rumelhart, D. E., \& Ortony, A. The representation of knowledge in memory. In R. C. Anderson, R. J. Spiro, \& W. E. Montague (Eds.), Schooling and the acquisition of knowledge. Hillsdale, N.J: Erlbaum, 1977.

RYLE, G. The concept of mind. London: Hutchinson, 1949.

Schank, R. C. Language and memory. Cognitive Science, 1980 , 4, 243-284.

Schank, R. C., \& Abelson, R. P. Scripts, plans, goals, and understanding. Hillsdale, N.J: Erlbaum, 1977.

Toglia, M. P., \& Battig, W. F. Handbook of semantic word norms. Hillsdale, N.J: Erlbaum, 1978.

Thonndike, E. L., \& Longe, I. The teacher's word book of 30,000 words. New York: Columbia University, Teachers College, Bureau of Publications, 1944.

Wollen, K. A., Weber, A., \& Lowry, D. H. Bizarreness versus interaction of mental images as determinants of learning. Cognitive Psychology, 1972, 3, 518-522.

(Manuscript received July 13, 1982;

revision accepted for publication February 9, 1983.) 